\title{
Monochromatic Electron Photoemission from Diamondoid Monolayers
}

\author{
W.L. Yang ${ }^{1,2}$, J.D. Fabbri ${ }^{1}$, T.M. Willey ${ }^{3}$, J.R.I. Lee $^{3}$, J.E. Dahl ${ }^{4}$, R.M.K. Carlson ${ }^{4}$, P.R. \\ Schreiner $^{5}$, A.A. Fokin ${ }^{5,6}$, B.A. Tkachenko ${ }^{5}$, N.A. Fokina ${ }^{5}$, W. Meevasana ${ }^{1}$, N. \\ Mannella ${ }^{1,2}$, K. Tanaka ${ }^{1,2}$, X.J. Zhou ${ }^{1,2}$, T. van Buuren ${ }^{3}$, M.A. Kelly ${ }^{1}$, Z. Hussain ${ }^{2}$, N.A. \\ Melosh $^{1}$, Z.-X. Shen ${ }^{1}$ \\ ${ }^{1}$ Geballe Laboratory for Advanced Materials, Stanford University, Stanford, CA 94305, USA \\ ${ }^{2}$ Advanced Light Source, Lawrence Berkeley National Laboratory, Berkeley CA 94720, USA \\ ${ }^{3}$ Lawrence Livermore National Laboratory, Livermore, California 94550, USA \\ ${ }^{4}$ MolecularDiamond Technologies, Chevron Technology Ventures, 100 Chevron Way, Richmond, \\ CA 94802, USA \\ ${ }^{5}$ Institut für Organische Chemie, Justus-Liebig University Giessen, Heinrich-Buff-Ring 58, 35392 \\ Giessen, Germany \\ ${ }^{6}$ Kiev Polytechnic Institue, pr. Pobedy 37, 03056 Kiev, Ukraine
}

We have found monochromatic electron photoemission from large-area self-assembled monolayers of a functionalized diamondoid, [121]tetramantane-6-thiol. Photoelectron spectra of the diamondoid monolayers exhibit a peak at the low kinetic energy threshold, with up to $68 \%$ of all emitted electrons emitted within this single energy peak. The intensity of the emission peak is indicative of diamondoids being negative electron affinity materials. With an energy distribution width of less than $0.5 \mathrm{eV}$, this source of monochromatic electrons may find application in a number of technologies such as electron microscopy, electron beam lithography, and field emission flat panel displays. 
Diamondoids are molecules with cage structures that can be superimposed with that of bulk diamond. These diamond-crystal cages are fused together and terminated by hydrogen. Adamantane is the smallest member, consisting of a single diamond cage, with tetramantane (higher diamondoid) constructed by four cages. Since the discovery of higher diamondoids in petroleum together with selective functionalization techniques (1-3), these materials have attracted interest because of the potential of combining the properties of diamond and nano-materials. In addition to the optical, mechanical, and thermal properties of bulk diamond, hydrogen-terminated diamond surfaces feature negative electron affinity (NEA), an important issue for the development of electron emitters (4). However, difficulties in emission uniformity, electron injection and electron transport have hindered the use of bulk diamond for this purpose (4). The primary challenge for electron emitters therefore remains to find a material that would realize uniformly large, highly efficient, and highly monochromatic electron emission. Diamondoids are an interesting candidate for electron emission, as they are essentially fully hydrogen-terminated diamond clusters. Indeed, recent Quantum Monte Carlo calculations predict NEA for diamondoids with the size up to $1 \mathrm{~nm}(5)$.

Large-area self-assembled monolayers (SAMs) of a functionalized diamondoid, [121]tetramantane-6-thiol $(2,3)$, were assembled on $\mathrm{Ag}$ or $\mathrm{Au}$ substrates $(6,7)$. Thin films of unsubstituted [121]tetramantane were also fabricated by evaporating 
tetramantane powder at $50-80^{\circ} \mathrm{C}$ onto cleaned $\mathrm{Au}$ substrates in situ. Photoemission Spectroscopy (PES) and Near Edge X-ray Absorption Fine Structure (NEXAFS) measurements were performed ( 6 ) to analyze electron emission properties and the molecular orientation of the SAMs. Several different samples with different bias voltages $(0-9 \mathrm{~V})$ were measured to check reproducibility. We observed obvious changes in the spectra after a certain period of beam exposure. All PES data shown here were collected at $30 \mathrm{~K}$ with less than 30 min of X-ray exposure.

The molecular orientation of the [121]tetramantane-6-thiol SAMs was characterized using polarization-dependent NEXAFS, a technique used extensively to study alkanethiolate SAMs (8-10). Figure 1A shows the total electron yield NEXAFS spectra of [121]tetramantane-6-thiolate on Au. The NEXAFS resembles spectra from bulk and gas-phase [121]tetramantane $(11,12)$, indicating a high-purity film of tetramantanethiol adsorbed on the surface. The angular dependence in the NEXAFS implies the tetramantanethiol forms well-ordered monolayers with a preferential orientation. Absorption intensity of a NEXAFS resonance from a C 1s orbital depends directly on alignment between the linearly-polarized incident x-rays and the transition dipole moment into a particular unoccupied orbital $(8,9)$. The angular dependence is simulated by summing over each atomic center with transition dipole moments oriented along the bonds $(8,9)$. These simulations agree with the experimental data (Fig. 1B) when the 
tetramantanethiol, with the z-axis of the molecule defined by the S-C bond, are tilted by $30+/-10^{\circ}$ from the surface normal (Fig. 1C) (6). Additionally, the affinity of the thiol to the metal leads to thiolate-bound monolayers $(7,13-15)$, confirmed by S $2 p$ core-level X-ray photoelectron spectroscopy (16) (Fig. S2).

The key results of this work are seen in the PES spectra of [121]tetramantane-6-thiol SAMs grown on $\mathrm{Ag}$ (Fig. 2A) and Au (Fig. 3A) substrates. An emission peak appears for both surfaces at about $1 \mathrm{eV}$ kinetic energy, the onset of the spectra at low kinetic energy. The intensity of the peak exceeds all the valence band features. For SAMs grown on Ag and $\mathrm{Au}$, the sharp peak comprises about $68 \%$ and $17 \%$ of the total electron yield, respectively. This peak intensity is several times stronger than that found for hydrogen-terminated diamond surfaces (e.g. 4, 17-19). Even with a logarithm plot (insets), one can still see a sharp feature instead of the typical exponential decay of secondary electrons in this energy range.

In order to ensure that this unusual electron emission originates from the diamondoid monolayers, we applied two different techniques to cover or remove the monolayer in situ. Coating the diamondoid $\mathrm{SAM}$ with one monolayer of $\mathrm{C}_{60}$, which was evaporated onto the SAM surface, caused the sharp emission feature to vanish (Fig. 2B). Note the valence band of the $\mathrm{C}_{60}$ covered surface is neither from tetramantanethiol nor from $\mathrm{C}_{60}(20)$, but 
the origin of these features is not clear at present. The diamondoid SAM was also removed by annealing a SAM sample to $550^{\circ} \mathrm{C}$ in situ. Given the thermal stability of conventional alkane thiol SAMs is roughly $70^{\circ} \mathrm{C}(21)$, this treatment should remove the diamondoid, and as shown in Fig. 2C, the low kinetic energy peak completely disappears after annealing (22).

To investigate the importance of a monolayer of functionalized diamondoid versus a thin film of diamondoid, we compared [121]tetramantane-6-thiol SAMs with [121]tetramantane films. Figure 3B shows the PES spectrum of the [121]tetramantane film. The spectrum shows a weak peak at low kinetic energy, in sharp contrast with the data for [121]tetramantane-6-thiol SAMs (Figs 2A and 3A). Two factors may contribute to this difference. One is the poor electron conductivity within the thicker films versus through the monolayers, and the other is the role that the thiol groups play for the SAM samples. Significantly, this result indicates that the strong electron emission does not occur solely from the diamondoid surface, but that the metal substrate is intimately involved in the process.

We have further confirmed that the sharp peak remains at the same energy with varying photon excitation energy (Fig. S3). This rules out the possibility of core-level excitations and suggests this sharp feature is not from electrons directly excited by 
photons, but from electrons accumulated at an intrinsic energy level of the molecules.

PES has been used widely for studying NEA materials, and a sharp feature at a low kinetic energy threshold is often evident in the spectra of NEA $(4,17-19,23,24)$. Thus, the emission peak presented in this work provides direct evidence that certain functionalized diamondoids are NEA materials, consistent with the recent diffusion Monte Carlo (DMC) calculations (5). Moreover, the calculated DMC band gap, about $7 \mathrm{eV}$ for tetramantane (5), is also consistent with the band gap estimated from our photoemission spectra, 6 to $8 \mathrm{eV}$ (25). As another verification of NEA, we evaporated slightly potassium (K) metal onto the SAM sample. In the PES of a K-covered [121]tetramantane-6-thiol SAM on Ag substrate, the sharp peak retains its high intensity, and occurs at the same energy position (Fig. S4). This is another indication of NEA because K-deposition onto a positive electron affinity semiconductor will lead to a shift of the low kinetic energy cutoff and strong enhancement of the secondary electron background.

On a typical NEA surface, electrons excited into unoccupied states relax to the bottom of the conduction band due to inelastic scattering, normally referred to as the "secondary cascade". A number of secondary electrons will then accumulate at the bottom of the conduction band. For a surface with positive electron affinity (true for 
almost all untreated semiconductor surfaces), these accumulated electrons cannot escape. For an NEA surface, these accumulated electrons can be emitted directly because the vacuum level lies below the bottom of conduction band. As a result, a peak will be observed at the low kinetic energy threshold in PES $(4,17-19,23,24)$.

However, on diamondoid SAM surfaces, there is only a single layer of diamondoid molecules. The detailed mechanism responsible for the highly monochromatic emission is unknown at this stage. Naively, one may consider that photoexcited electrons lose energy by creating phonons in the molecules, but this will likely lead to the destruction of the molecules. A plausible scenario is that the majority of the photoexcited electrons come from the substrate. These electrons first thermalize in the metal, producing many more low energy electrons. Electrons with energies above the diamondoid conduction band minimum may get transferred to diamondoid molecules, reach the bottom of the conduction band by creating phonons and get emitted. This proposal is shown schematically in Fig. 4. The other difference of our results with that of other typical NEA systems $(4,17-19,23,24)$ is that our data show a spike in the spectra rather an exponential rise of the secondary tail towards the threshold, suggesting that a single energy level, resulted from the molecular nature of nanometer sized diamondoids, and/or a strong resonance process are involved. 
Our results suggest that diamondoid monolayers may have promising utility. Not only can functionalized diamondoids be easily grown into large area SAMs with NEA properties, they naturally circumvent the long standing electron-conductivity issues encountered for wide-gap bulk NEA semiconductors $(4,26)$. On a diamondoid SAM surface, electron conduction from the electron reservoir (metal substrates) to the emission surface is through one single molecule, which successfully avoids the low conductivity problem and enhances the election emission. Additionally, the possibility of different functionalizations $(3,4)$ provides us an opportunity to optimize the NEA and other properties of diamondoids. While many technical issues need to be addressed for utilizing diamondoid SAMs as electron emitters, the molecular nature of diamondoids provides intrinsic advantages over bulk materials because of their special molecular characteristics; e.g. narrow energy distribution of the electronic states - an interesting problem requiring more study.

\section{REFERENCES \& NOTES}

1. J. E. Dahl, S. G. Liu, R. M. K. Carlson, Science 29996 (2003).

2. B. A. Tkachenko et al., Organic Letters 81767 (2006).

3. P. R. Schreiner et al., J. Org. Chem. 718532 (2006).

4. For a review, The Physics of Diamond: Proceedings of the International School of Physics. Edited by A. Paoletti and A. Tucciarone, (IOS press, 1997). 
5. N. D. Drummond, A. J. Williamson, R. J. Needs, G. Galli, Phys. Rev. Lett. 95096801 (2005).

6. Materials and methods are available as supporting material on Science Online.

7. A. Ulman, Chem. Rev. 96, 1533 (1996).

8. J. Stöhr, NEXAFS Spectroscopy, (Springer-Verlag, 1992).

9. G. Hähner, Chemical Society Reviews 35 1244-1255 (2006).

10. M. Zharnikov, M. Grunze, Journal of Physics-Condensed Matter 13 11333-11365 (2001).

11. T. M.Willey et al., Phys. Rev. Lett. 95113401 (2005).

12. T. M. Willey et al., Phys. Rev. B 74, 205432 (2006).

13. P. E. Laibinis et al., J. Am. Chem. Soc. 1137152 (1991)

14. A. Shaporenko et al., Langmuir 214370 (2005).

15. J. C. Love et al., Chem. Rev. 1051103 (2005).

16. D. G. Castner, K. Hinds, D. W. Grainger, Langmuir 125083 (1996).

17. F. J. Himpsel, J. A. Knapp, J. A. VanVechten, D. E. Eastman, Phys. Rev. B 20, 624 (1979).

18. B. B. Pate Surf. Sci. 16583 (1986).

19. J. van der Weide, R. J. Nemanich Appl. Phys. Lett. 621878 (1993).

20. W. L. Yang et al., Science 300303 (2003).

21. C. D. Bain et al., J. Am. Chem. Soc. 111, 321, 1989 
22. The fact that we did not see a strong secondary electron component on the annealed sample may indicate that the transmission efficiency of the spectrometer is poor at low kinetic energies. This suggests that the peak from SAM surface could have been even stronger. Artificial effects on the peak have been ruled out by testing with different bias voltages.

23. L. W. James, J. L. Moll, Phys. Rev. 183740 (1969).

24. R. C. Eden, J. L. Moll, W. E. Spicer, Phys. Rev. Lett. 18, 597 (1967).

25. For NEA materials, the difference between photoemission spectral width and the excitation energy should match the band gap value (4, 17-19). Although there is no precise gap value reported for [121]tetramantane-6-thiol, and it is difficult to define a precise photoemission spectral width of an insulating monolayer system due to the difficulty in determining the spectral onset, the estimation from our spectra is consistent with the DMC calculation (5), considering the thiol groups are likely to change the gap value by only few tens of an $\mathrm{eV}$.

26. R. L. Bell, Negative electron affinity devices (Clarendon Press, Oxford, 1973).

27. Acknowledgement: We acknowledge helpful discussions with Zhi Liu, Dung-Hai Lee, and Howard Padmore. YWL thanks Yayu Wang for sharing information on diamondoid film deposition, and John Pepper, Scott DiMaggio for technical support. The work at SSRL and ALS is supported by the DOE Office of Basic Energy Science, Division of Material Science, under contracts DE-FG03-01ER45929-A001 
and DE-AC03-765F00515, respectively. The work at Stanford is also supported by Chevron through the Stanford-Chevron Program on Diamondoid Nano-Science. 


\section{FIGURE CAPTIONS}

Figure 1. (A) Polarization-dependent total electron yield NEXAFS spectra collected on [121]tetramantane-6-thiol SAMs prepared on Au. Total electron yield is plotted against photon energy at different beam incident angles. The leading edge peak at $287.6 \mathrm{eV}$ (red oval) is assigned to transitions from the $\mathrm{C} l s$ core level to the unoccupied $(\mathrm{C}-\mathrm{H}) \sigma^{*}$ orbitals, while the peak at about $292.5 \mathrm{eV}$ is assigned to (C-C) $\sigma^{*}$ orbitals $(8,10,11)$. The second gap indicated by the arrow is the characteristic signature of diamondoids $(11,12)$. (B) Comparison between experiments and theoretical simulations. Red squares represent the experimental ratio of $(\mathrm{C}-\mathrm{H}) \sigma^{*}$ spectral weight between data at different angles and that at $20^{\circ}$. The black line is the calculated ratio based on the molecular geometry as shown in (C), with polar angle of $36.5^{\circ}(6)$.

Figure 2. (A) Photoelectron spectra of [121]tetramantane-6-thiol SAMs grown on Ag substrates, collected with $55 \mathrm{eV}$ photon energy. The strong peak at $1 \mathrm{eV}$ kinetic energy contains $68 \%$ of the total photoelectrons. The dotted line is a 50 times blowup of valence band features. The inset shows the same spectra in a double logarithm plot. (B) Photoelectron spectrum collected on [121]tetramantane-6-thiol SAM covered by $\mathrm{C}_{60}$ sublimed in situ. The strong electron emission peak disappears after $\mathrm{C}_{60}$ coverage. (C) Photoelectron spectrum collected on an annealed [121]tetramantane-6-thiol SAM. Again, the peak observed for the pristine SAM vanishes after the in situ annealing to $550^{\circ} \mathrm{C}$. The 
difference between Fig. 2c and a pure Ag PES spectrum could be partially due to some residual sulfur atoms still bounded to the surface after annealing.

Figure 3. (A) Photoelectron spectra of [121]tetramantane-6-thiol SAMs grown on $\mathrm{Au}$ substrates. The sharp peak at $1 \mathrm{eV}$ contains about $17 \%$ of the total photoelectrons. The inset is a double logarithm plot. (B) Photoelectron spectra of unsubstituted [121]tetramantane films prepared in situ on Au substrates. The inset is a blowup of the low kinetic energy part with only a weak peak.

Figure 4. Schematic of the electron emission process on diamondoid SAM surfaces. $\mathrm{E}_{\mathrm{F}}$ is the Fermi level of the metal substrate, sitting in the energy gap of diamondoid. The vacuum level ( $\left.\mathrm{E}_{\text {Vacuum }}\right)$ is below the conduction band minimum of the diamondoid, a characteristic of NEA. The dotted red line depicts the high probability electron emission. Firstly, electrons in metal substrates are excited by photons into unoccupied states above $\mathrm{E}_{\mathrm{F}}$. Secondly, the excited electrons effectively thermalize in the metal, producing more electrons with lower energy. Thirdly, electrons with energy above the conduction band minimum are transferred to diamondoid moieties. These electrons further lower their energies by exciting phonons in the molecules, and accumulate at the bottom of conduction band. Finally, due to NEA, electrons accumulated at the bottom of conduction band emit into vacuum spontaneously and generate a peak at the low kinetic energy 
threshold. There is also electron emission taking place at high kinetic energy levels, but with much lower photoelectron yield. Although this scenario roughly explains the existence of the electron emission peak, more theoretical inputs are needed to fully understand the results. 


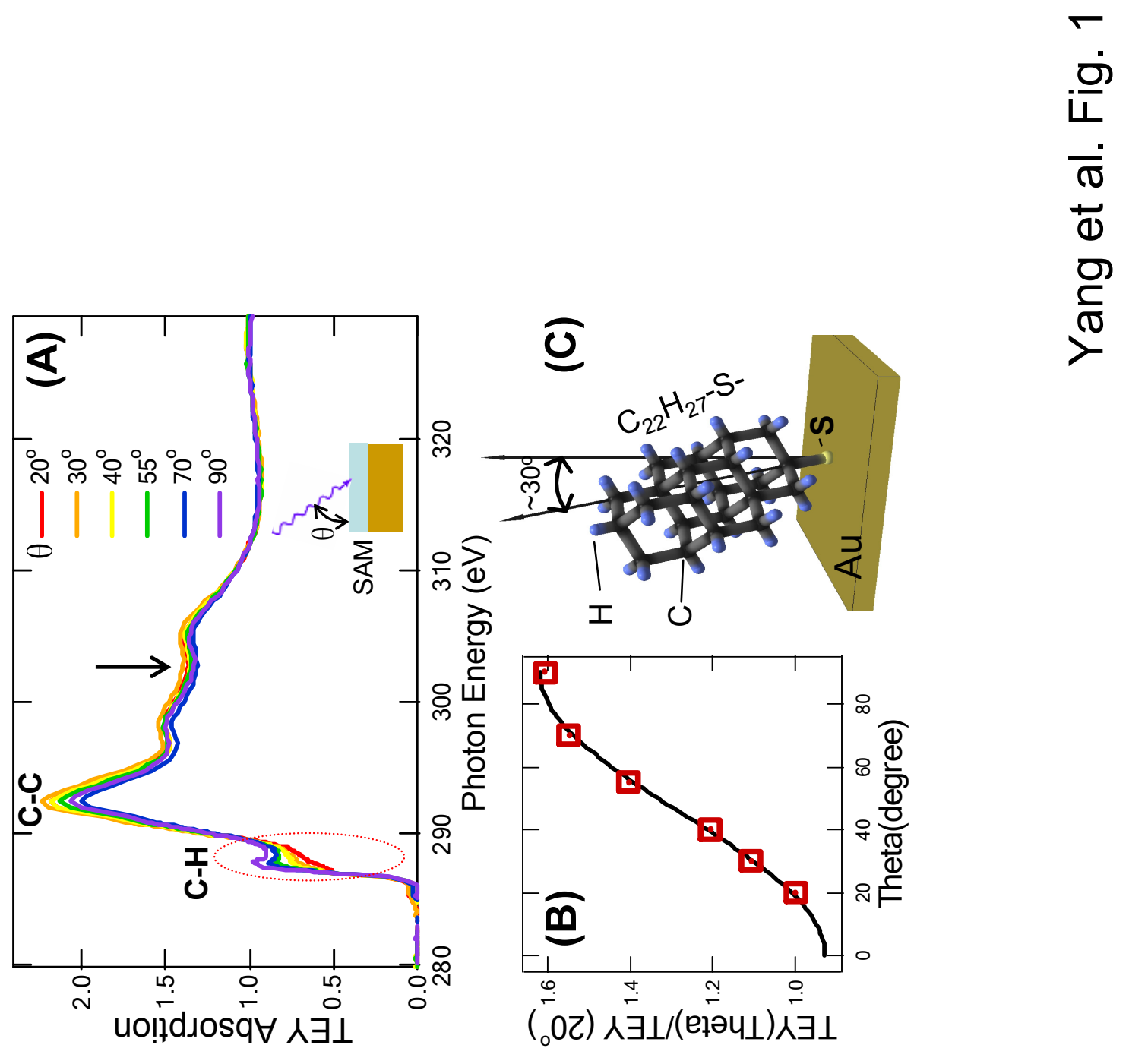




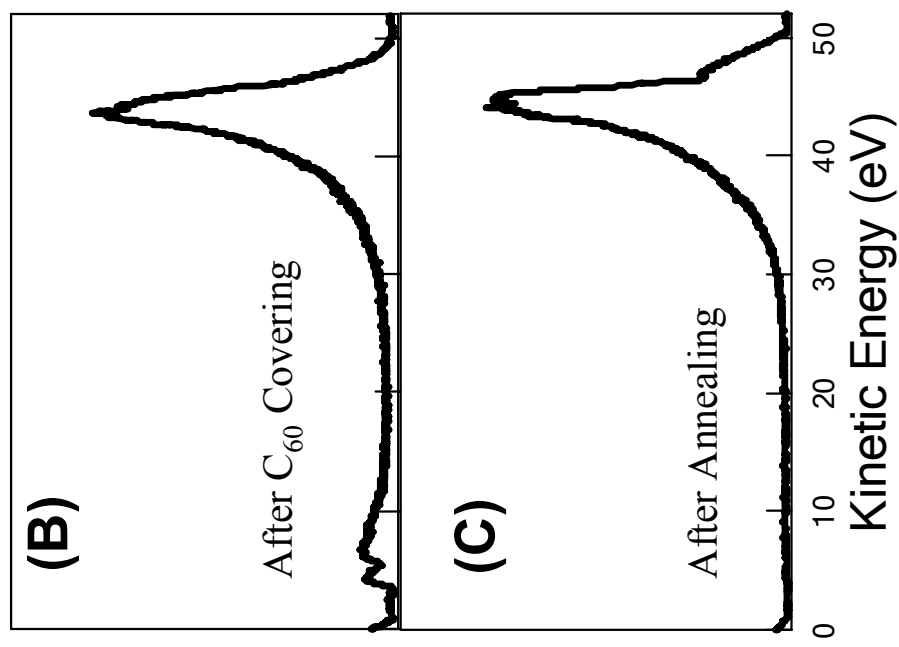

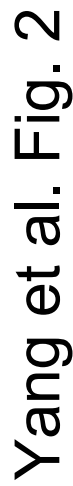

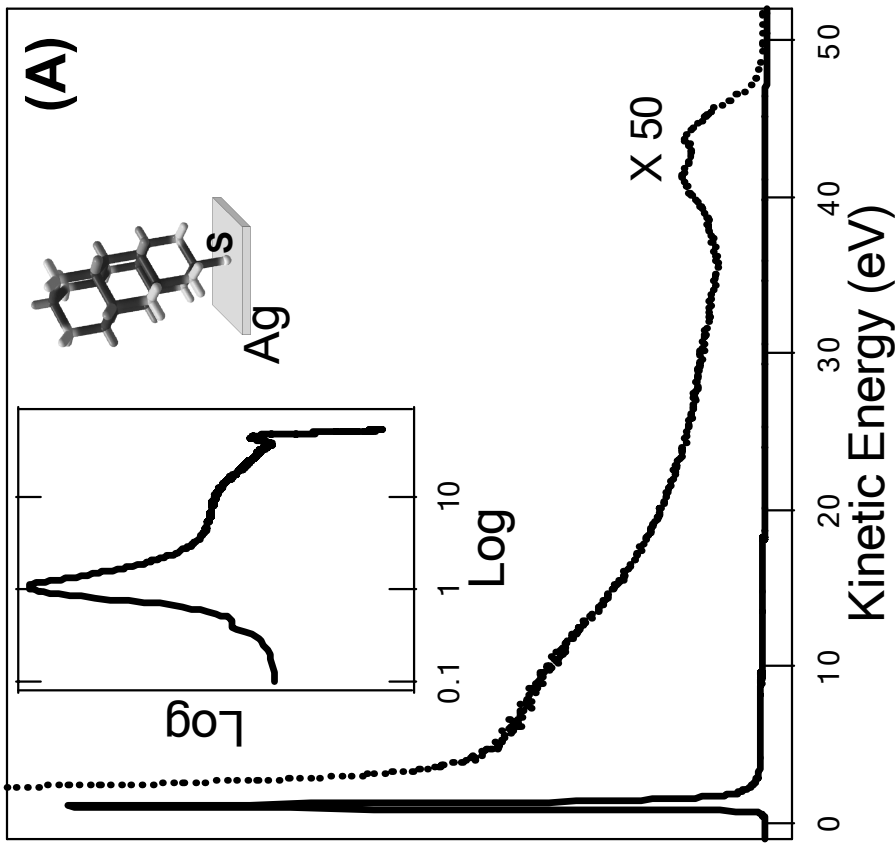

(sı!un 'qл 


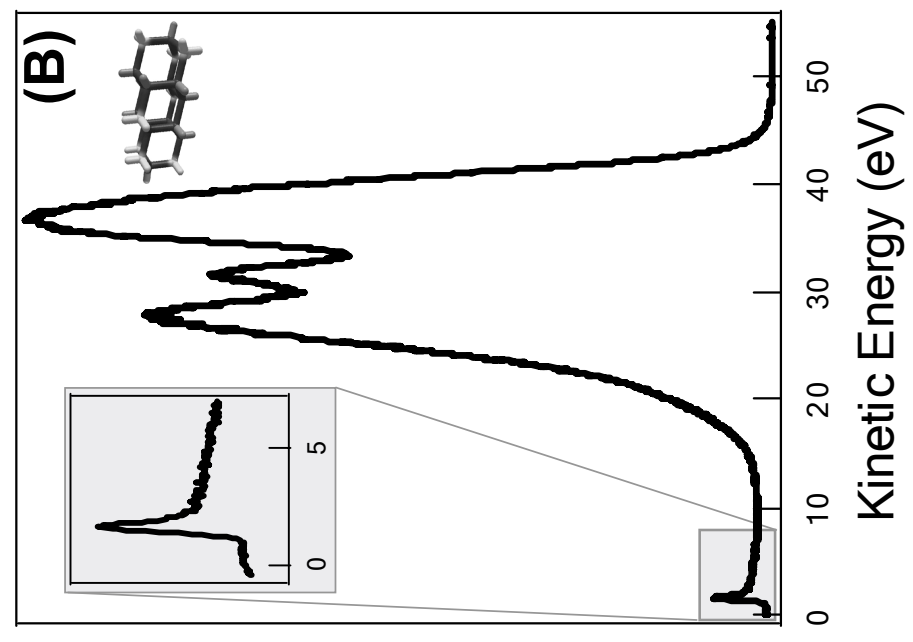

$m$
$\dot{0}$
$\frac{0}{4}$
$\dot{\sigma}$
$\frac{1}{0}$
$\frac{0}{\sigma}$
$\frac{0}{\sigma}$

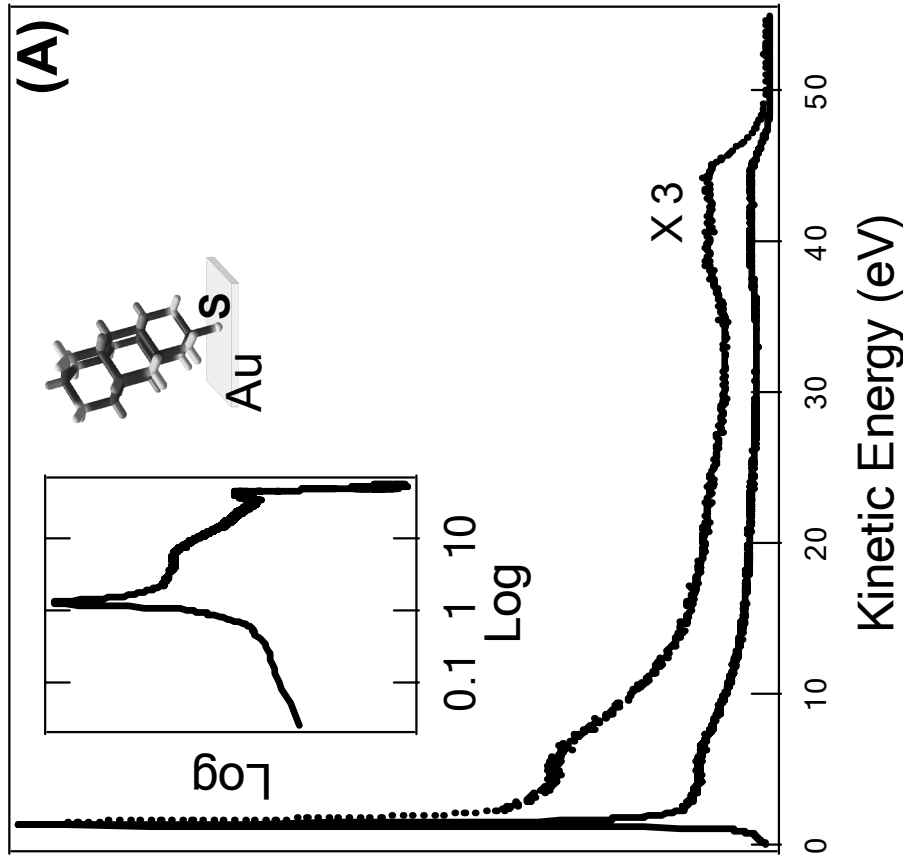

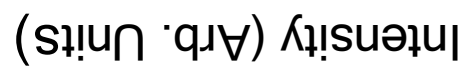




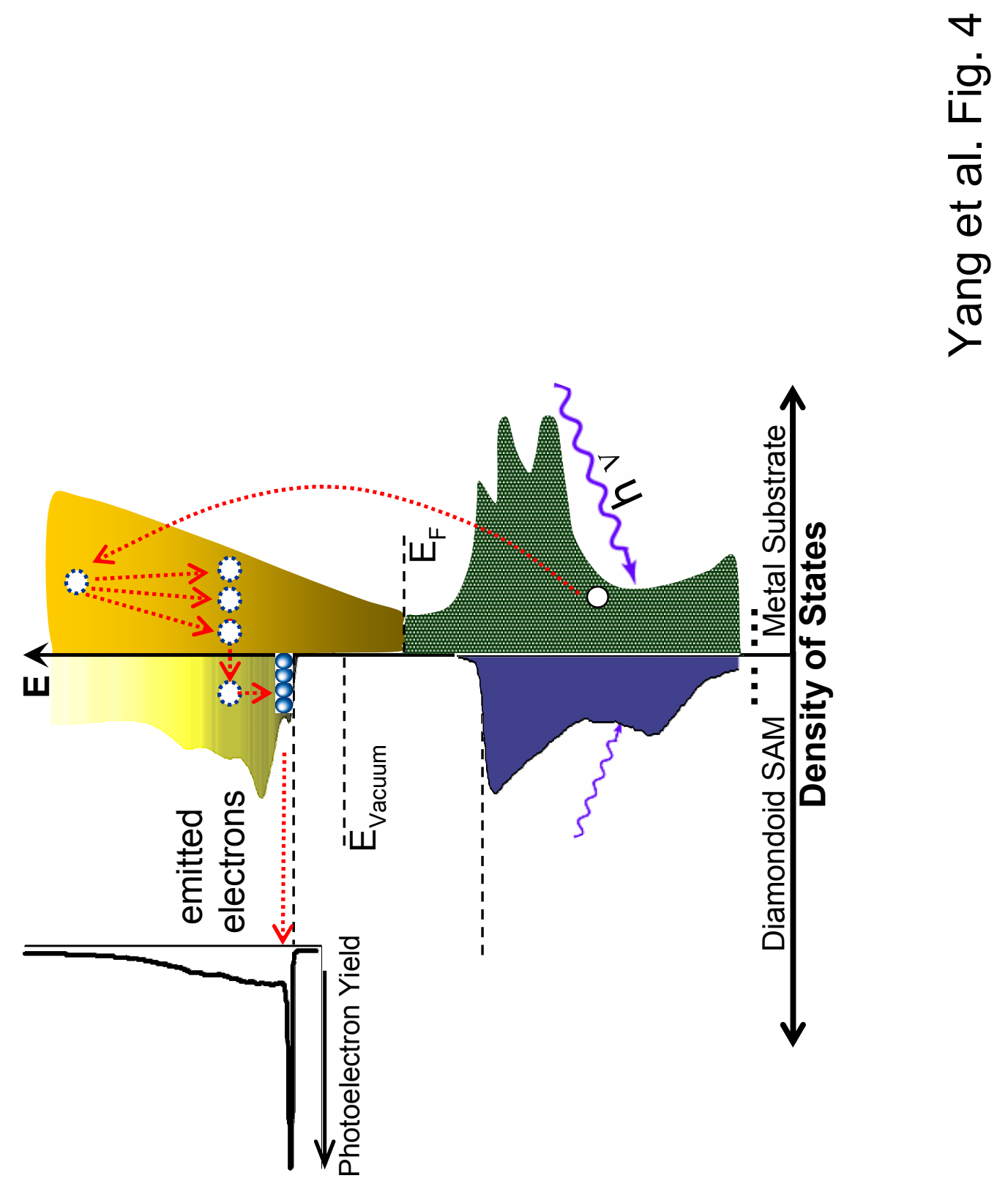




\section{Supporting Online Material}

\section{Materials and Methods}

\section{Sample preparation}

SAMs of [121]tetramantane-6-thiol were prepared in solution at room temperature via self-assembly from ethanol/toluene solution. For SAMs grown on Au, a layer of $3 \mathrm{~nm}$ Ti followed by $100 \mathrm{~nm} \mathrm{Au} \mathrm{was}$ deposited by e-beam evaporation at a base pressure of approximately $1 \times 10^{-6}$ torr onto either $\mathrm{Au}$ or $\mathrm{Ag}$ substrates with the size of approximately $1 \mathrm{~cm}^{2}$. The SAMs were then grown by immersing the metal surfaces into the solutions for one to two days. Upon removal from the solution, the films were vigorously washed with toluene and ethanol under an $\mathrm{N}_{2}$ atmosphere and immediately loaded into the vacuum chamber.

\section{Photoelectron spectroscopy (PES)}

PES data were collected by using a SCIENTA R4000 electron spectrometer at the HERS endstation, BL10.0.1, Advanced Light Source (ALS). Before the exposure to the synchrotron beam, the SAMs were very gently degassed, sometimes by annealing to about $30^{\circ} \mathrm{C}$. The linear polarization of the incident beam is $20^{\circ}$ off the sample surface. All data were taken with pass energies as low as $2 \mathrm{eV}$, and samples were biased from 0 to -9 volts to exceed the spectrometer work function as well as to check the reliability of the low kinetic energy data. We observed obvious changes in the spectra after a certain period of beam exposure. All PES data shown here were collected at $30 \mathrm{~K}$ with less than 30 minutes of X-ray exposure; no sign of radiation damage on PES spectra was detected under this condition.

\section{Near Edge X-ray Absorption Fine Structure (NEXAFS) experiments}

NEXAFS spectra were recorded at beamlines 8.2 and 10.1 of the Stanford Synchrotron Radiation Laboratory (SSRL, SPEAR III) at the Stanford Linear Accelerator (SLAC). NEXAFS spectra were recorded using Total Electron Yield (TEY), obtained by measuring the total current leaving the experimental sample as the X-ray energy was scanned across the carbon absorption edge. The NEXAFS 
signals were normalized to the incident photon flux, as recorded by the TEY signal of a freshly gold coated grid in the beam. The two major angular-dependent resonances $\left(\mathrm{R}^{*} / \mathrm{C}-\mathrm{H} \sigma^{*}\right.$ and the $\left.\mathrm{C}-\mathrm{C} \sigma^{*}\right)$ were used in determining the orientation of the molecules, but only the results on $\mathrm{R}^{*} / \mathrm{C}-\mathrm{H} \sigma^{*}$ are shown here.

\section{NEXAFS simulations}

[121]Tetramantane has sufficient majority of the bonds that are oriented, in general, close to orthogonal (C-H) and normal (C-C) to the long molecular axis. This allows determination of molecular orientation. As another typical way to present the NEXAFS polarization dependence, Fig. S1A shows the difference of the spectral weight at various beam incident angles relative to the spectrum acquired at $20^{\circ}$. The same data is plotted in Fig. 1A.

By simple inspection, the spectra acquired at normal incidence $\left(90^{\circ}\right)$ exhibits the strongest intensity for the $\mathrm{R}^{*} /(\mathrm{C}-\mathrm{H}) \sigma^{*}$ peak, meaning the $(\mathrm{C}-\mathrm{H})$ orbitals are oriented more prostrate than normal with respect to the surface. Alternatively, the $(\mathrm{C}-\mathrm{C}) \sigma^{*}$ resonance shows the opposite angular dependence, meaning the $(\mathrm{C}-\mathrm{C})$ bonds are generally oriented more normal to the surface than prostrate. Thus, the molecule is upright on the surface. The general, gross approximation of summing the transitions dipoles oriented along the respective $\sigma^{*}$ resonances from each atomic center in the molecule was used $(S 1)$ to determine the polar angle of the molecule with respect to the surface. Although this model is an approximation (S2-S4), it allows a more quantitative estimate of the orientation.

This method is described in detail in $(S 1)$, but for completeness and for details specific to [121]tetramantane-6-thiolate, a brief summary is provided here. Firstly, the NEXAFS spectra at all incidence angles were fit using the method described elsewhere (S5) to deconvolute angular dependent $\mathrm{R} * /(\mathrm{C}-\mathrm{H}) \sigma^{*}$ and $(\mathrm{C}-\mathrm{C}) \sigma^{*}$ resonances. The intensity of a resonance is proportional to square of the dot product of the electric field and the transition dipole moment, which is along the final state orbital for excitations from a 1s core-level, using the dipole approximation $(S 1)$. Secondly, ratios of intensities are considered to remove the proportionality that is due to factors like detector efficiency and transition cross section. Figure S1B depicts the experimental ratio, $\mathrm{I}(\theta) / \mathrm{I}\left(20^{\circ}\right)$, of the reliable $\mathrm{R}^{*} /(\mathrm{C}-\mathrm{H}) \sigma^{*}$ 
resonances from two different samples (red and blue dots). The analysis returns $26+/-3$ and $36.5+/-4$ respectively. Thirdly, we also consider a myriad of potential systematic errors such as beamline stability and polarization variation, fitting errors, subtle sample-to-sample variation, as well as the dipole approximation and other assumed approximations (S2-S4). In light of these systematic errors, we estimated +/- 10 as the error bar for the polar angle of the molecule on the surface.

Twist or dihedral angle often also plays a role, but in this case, $30+/-10$ degrees is valid with dihedral angles to about $+/-100^{\circ}$ with the geometry chosen above. At greater dihedral angles, simulation of the NEXAFS returns polar angles that are no longer sterically possible, i.e. physically impossible orientations in which atomic positions in the molecule are now below the gold surface plane. Thus, the polar angle of the molecule is $30+/-10$, but with this method, the dihedral (twist) angle about the S-C bond can not be uniquely determined. 

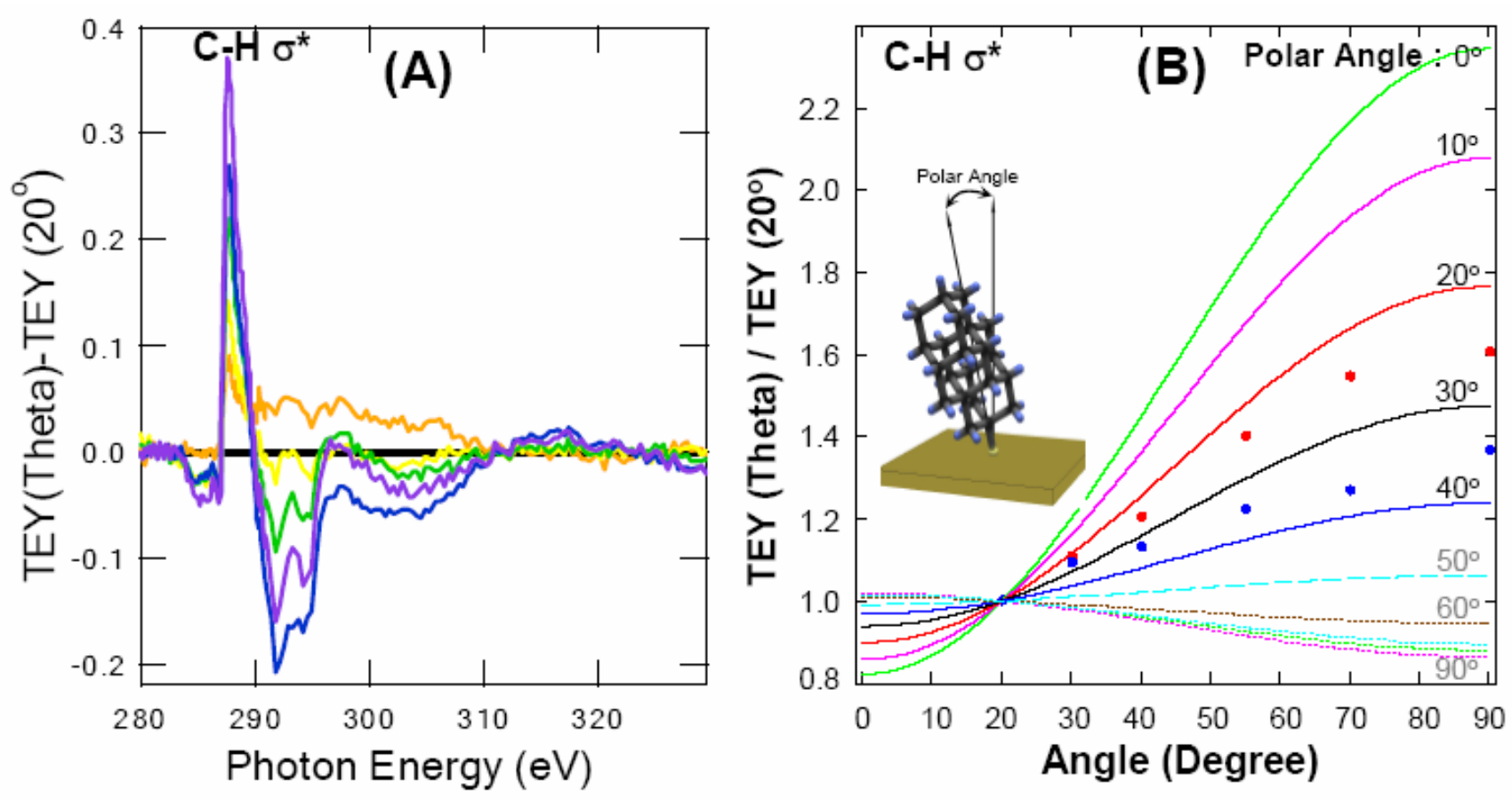

Figure S1. (A) Difference of the spectral weight at various beam incident angles relative to the spectrum acquired at $20^{\circ}$. There is a clear variation of the $(\mathrm{C}-\mathrm{H}) \sigma^{*}$ peak at $287.6 \mathrm{eV}$ upon the beam incident angles. The raw data was shown in Fig. 1A. (B) Red and blue dots are experimental ratio of the spectral weight at various beam incident angles to that at $20^{\circ}$, collected on two samples. Lines are calculated ratio based on different molecular polar angle, as defined by the $\mathrm{S}-\mathrm{C}$ bond direction (inset). The red dots are also plotted in Fig. 1B to show a good agreement with the calculation based on polar angle of $36.5^{\circ}$. The dashed and dotted lines are unphysical orientations of the molecule (see SOM, NEXAFS simulations). 


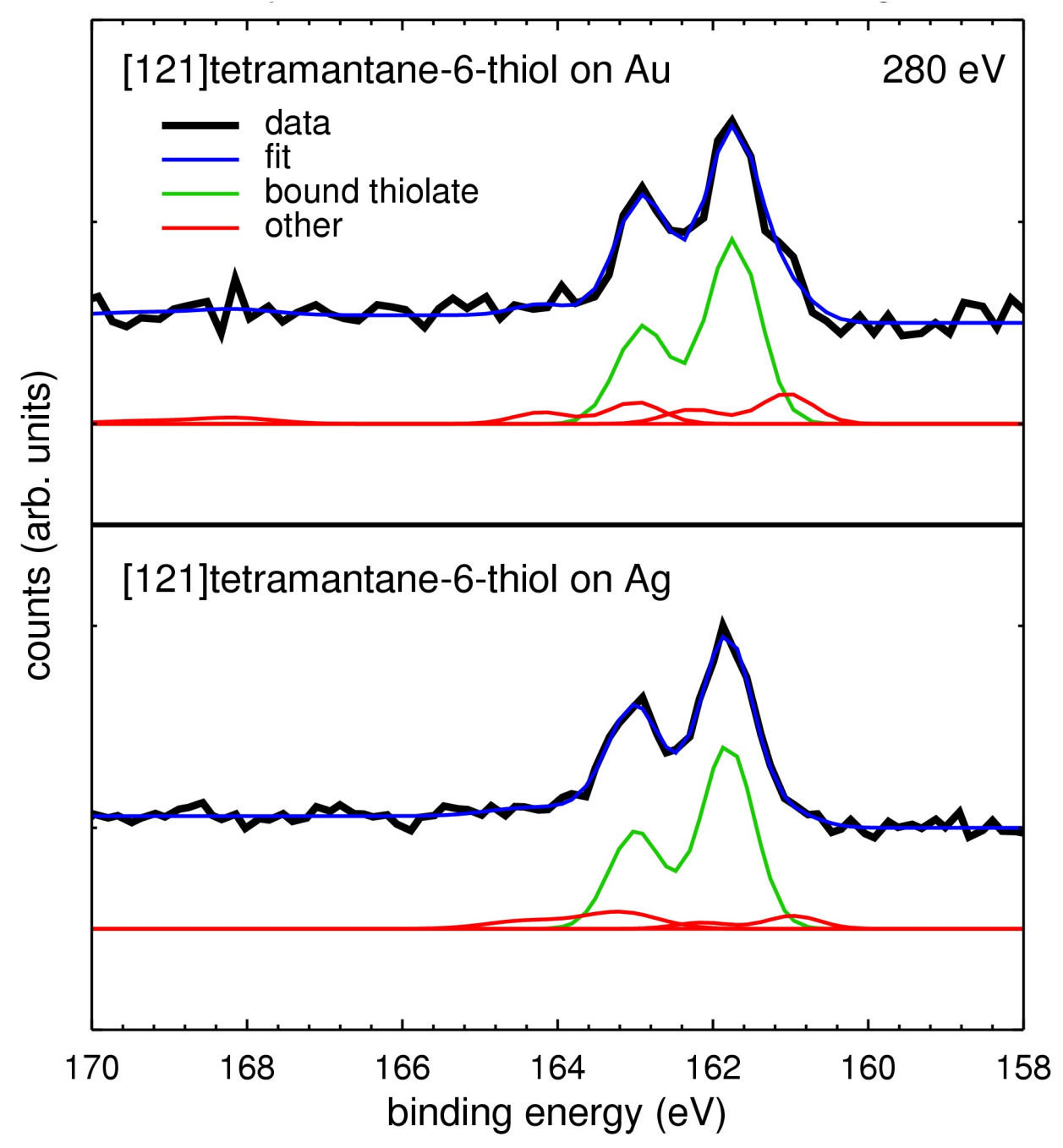

Figure S2. Black lines are $\mathrm{S} 2 p$ core-level X-ray photoelectron spectra (XPS) of [121]6-tetramantanethiol SAMs on Au (top panel) and Ag (bottom panel). Blue lines are fittings to the data based on Au-thiolate and Ag-thiolate sulfur (gree lines). Other S species, unbound, elemental, and oxidized sulfur, only have insignificant contributions (red lines). 


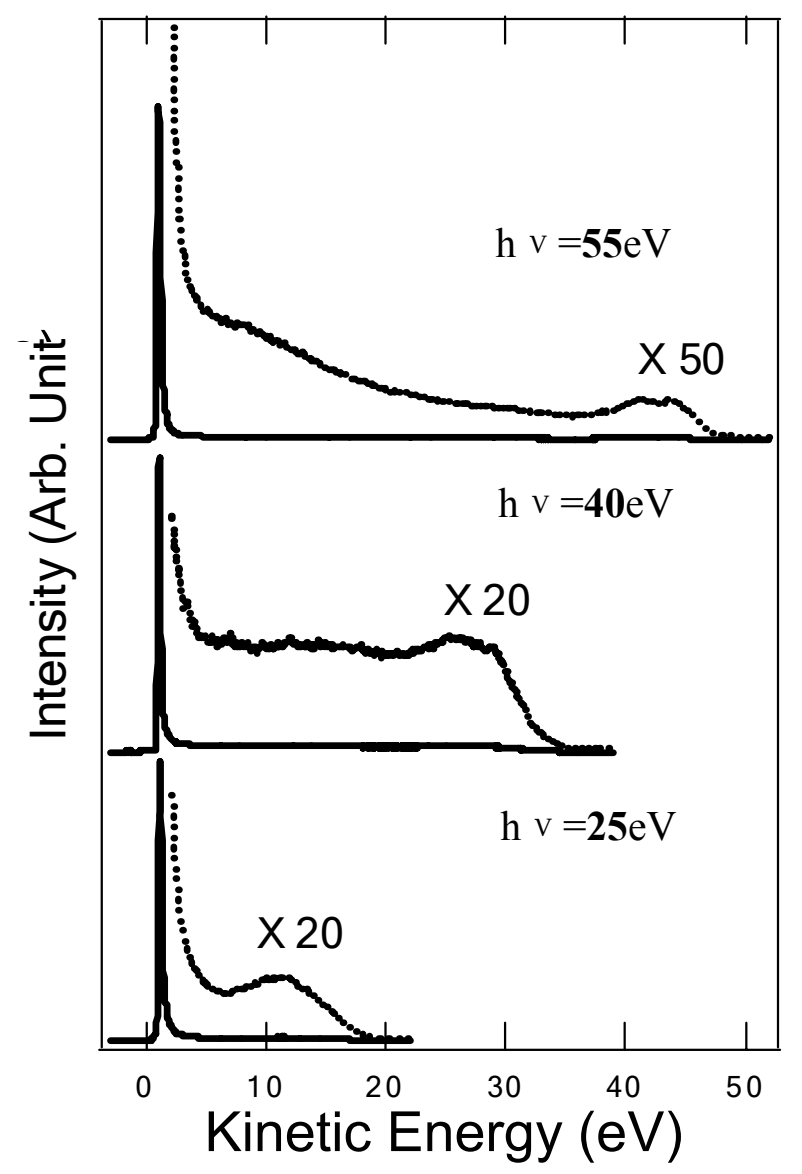

Figure S3. Photoelectron spectra of [121]6-tetramantanethiol SAMs on Ag collected with $25 \mathrm{eV}, 40 \mathrm{eV}$, and $55 \mathrm{eV}$ photon energy. The unusual electron emission peak at $1 \mathrm{eV}$ persists. A quantitative analysis of the peak intensity upon photon energy is non-trival because of the unavoidable higher harmonic components of synchrotron photon beam. 


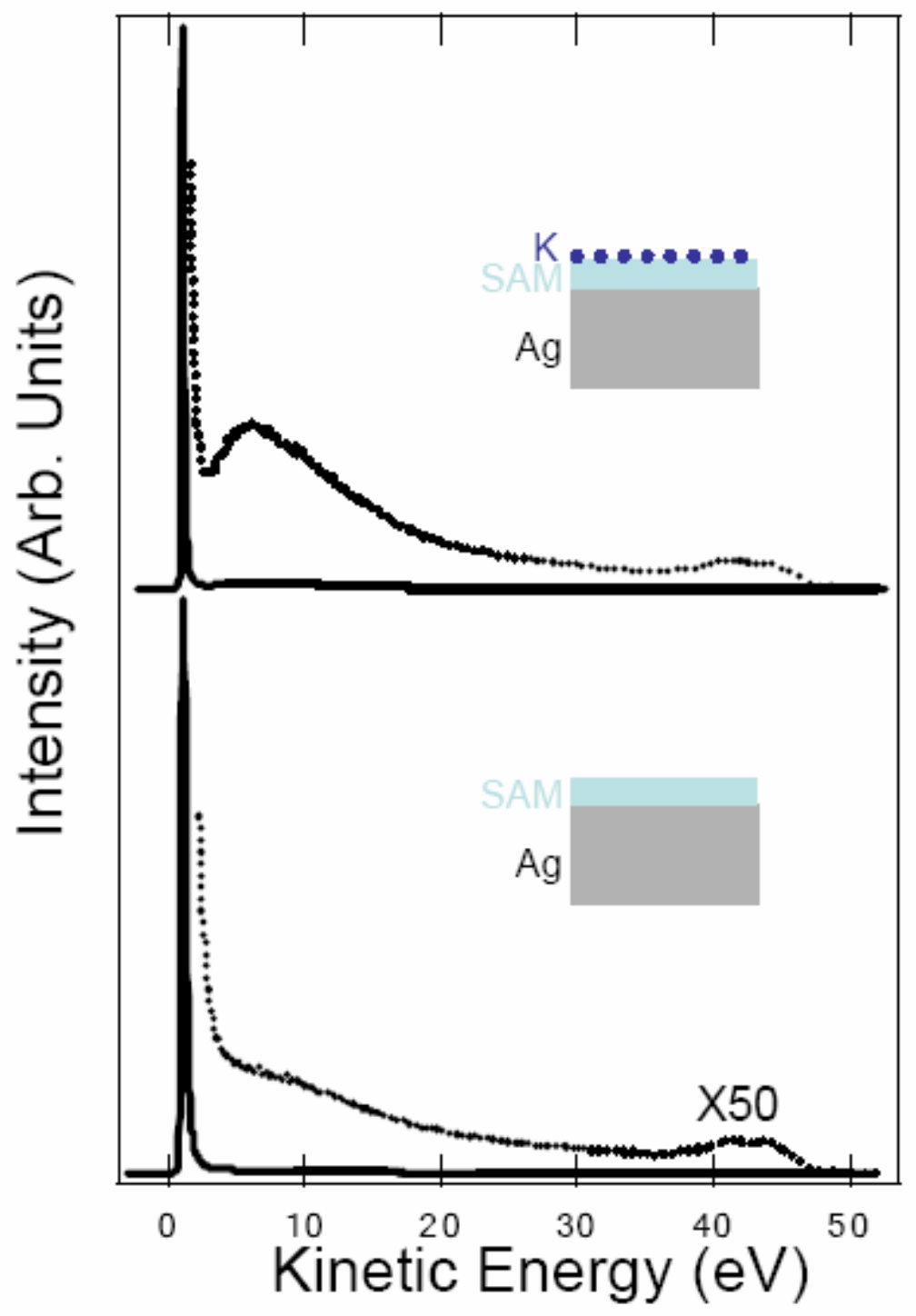

Figure S4. Photoelectron spectra of [121]6-tetramantanethiol SAMs with (upper) and without (lower) potassium deposition. No energy shift was observed for the sharp peak, and only a small increase of the secondary electron background can be seen. 


\section{Supporting references}

S1. J. Stöhr, NEXAFS Spectroscopy, Springer-Verlag, 1992

S2. G. Hähner, et al., Phys. Rev. Lett. 67, 851 (1991).

S3. J. X. Fu and S. G. Urquhart, J. Phys. Chem. A 10911724 (2005).

S4. Y. Zou et al., Chem. Phys. Lett. 430287 (2006).

S5. T. M. Willey, Langmuir, 20, 2746 (2006); and online supporting information 